\title{
El monumento a las banderas en la avenida de las Américas en Bogotá Monumento a las Banderas on Avenida de las Américas in Bogota
}

Diego Carrizosa Posada (Colombia)

Institución Universitaria Politécnico Grancolombiano correo: diegocar@poli.edu.co

\section{Resumen}

El presente ensayo tiene como objetivo indagar sobre el momento sociopolíitico que se vivió en el país desde 1946 hasta 1948 y por qué razón se encarga al artista Alonso Neira Martínez la realización arquitectónica y escultórica del monumento a las banderas; conocer las políticas de diseño urbano que durante esos años imperaron en la capital y de qué manera beneficiaría a los bogotanos la construcción de la avenida de las Américas; asimismo,

\begin{abstract}
This essay has three objectives, the first one is to find out about the sociopolitical moment lived in Colombia from the years 1946 to 1948. The second objective concentrates to know the urban political design normativity valid in Bogota from 1946 a 1948, and how the construction of the Avenue of the Americas will benefit the citizens of the capital. And the last one goes to query about the biography and the reason why Alonso Neira Martínez (1913-1991), chooses to design in
\end{abstract}


conocer la biografía del artista Alonso Neira Martínez (1913-1991) e indagar sobre las razones por las cuales realizó un diseño escultórico neoclásico en el monumento a las banderas.

Palabras claves: monumento a las banderas, avenida de las Américas, Alonso Neira Martínez. a neo classical way the monumento a las Banderas.

Keywords: monumento a las banderas, Avenue of the Americas, Alonso Neira Martínez. 


\title{
El monumento a las banderas en la avenida de las Américas en Bogotá
}

\author{
Diego Carrizosa Posada (Colombia) \\ Profesional en Producción y Dirección de Cine y Televisión por el Columbia College, \\ de Los Ángeles, Estados Unidos. Fue galardonado con el Premio Nacional de \\ Periodismo Simón Bolívar, otorgado a la serie Un día en la vida de... como el \\ Mejor Programa Cultural, en 1987. Actualmente es profesor en el Departamento \\ de Medios Audiovisuales del Politécnico Grancolombiano y adelanta estudios de \\ Maestría en Estética e Historial del Arte en la Universidad Jorge Tadeo Lozano.
}

El monumento a las banderas, obra realizada por el artista bogotano Alonso Neira Martínez en 1948, será el tema principal para indagar en este ensayo. Buscaré conocer y contextualizar con el conjunto escultórico lo acontecido en el país en la segunda mitad de los años cuarenta en el ámbito artístico, social, político y económico. El objeto de estudio de este ensayo corresponde a varios puntos:

- Analizar el momento sociopolítico que se vivió en el país desde 1946 hasta 1948 y por qué razón se encargó al artista Alonso Neira Martínez la realización arquitectónica y escultórica del monumento a las banderas.

- Conocer las políticas de diseño urbano que entre 1946 y 1948 imperaron en la capital y de qué manera beneficiaría a los bogotanos la construcción de la avenida de las Américas.

- Conocer la biografía del artista Alonso Neira Martínez (1913-1991) e indagar sobre las razones por la cuales realizó un diseño escultórico neoclásico en el monumento a las banderas. 
Mariano Ospina Pérez ejerció la Presidencia de Colombia desde 1946 hasta 1950, llegó al poder con el lema de "la Unión Nacional", en el que se consigna que no habría persecución política contra las personas ni contra sus bienes, por parte de las autoridades, ni exclusiones al servicio administrativo por motivos partidistas; se garantizaría fielmente el ejercicio de los derechos naturales y civiles y se harían respetar celosamente las libertades políticas (Meiseles, 1992, p. 286). Ospina Pérez inició su gobierno con varias huelgas y paros laborales, entre los que se destacan el originado en la Federación de Trabajadores del Valle, la Unión Sindical Obrera (USO) y la Tropical Oil Company de Barrancabermeja. En 1946 el Congreso, de mayoría liberal, vetó la colaboración de participantes de su filiación política en el gabinete ministerial. Jorge Eliécer Gaitán hizo lo mismo, investido por el poder obtenido como resultado de sus logros en las elecciones para corporaciones públicas y como respuesta a las promesas fallidas en torno a las persecuciones políticas ejercidas contra miembros de su partido; sin embargo, esta colaboración se prestó hasta 1948. Entre 1947 y 1949 gigantes industriales como Coltejer, Fabricato, Colombiana de Tabaco, Cementos Diamante, Bavaria y Azucarera del Valle presentaron utilidades del $200 \%$. En ese momento la situación económica de la clase obrera colombiana (mano de obra primaria y coproductora de esas ganancias), era de extrema dificultad, por cuanto durante dicho periodo los elementos de primera necesidad duplicaban o triplicaban sus precios. Además, el país estaba siendo afectado de manera importante por situaciones, como una alta especulación en los precios de la canasta familiar, el tráfico de influencias y el incremento de los valores de los arriendos, a consecuencia de una decisión equivocada de inversión de divisas en finca raíz por parte del Gobierno. Como resultado de lo anterior, el país se encontraba en una difícil situación social, política y económica. La represión laboral se acrecentó en la administración de Ospina Pérez, la violencia oficial afectó a campos y ciudades y miles de vidas pertenecientes a ambos partidos se perdieron a causa del sectarismo político. Gaitán protestó en Bogotá contra esta ola de violencia desmesurada y el 7 de febrero de 1948, ante una gran cantidad de colombianos, en la plaza de 
Bolívar pide "que haya paz y piedad por la patria". Posteriormente, el 30 de marzo de 1948, se inauguró en la capital la IX Conferencia Panamericana, con la asistencia de los representantes de veintiuna naciones, que en ese momento conformaban el continente americano.

El objetivo de esta asamblea era doble: consolidar a la Organización de Estados Americanos (OEA) como un órgano legal con clara ascendencia sobre los Estados americanos, y evitar la injerencia del comunismo en América. Para conmemorar este importante acontecimiento Bogotá se había renovado con obras públicas de enlucimiento, como el monumento a las banderas. La fecha escogida para celebrar la asamblea, así como para inaugurar el monumento de Neira Martínez, correspondía al 9 de abril de 1948. De repente, cuando todo marchaba normalmente, al salir de su oficina, situada en el edificio Nieto, Gaitán fue asesinado, y como el país conoce, los partidarios del líder inmolado reaccionaron y una ola desbordada de violencia se tomó la capital. Bogotá sufrió la pérdida de aproximadamente tres mil ciudadanos y quedó semidestruida, con daños irremediables en su patrimonio cultural y arquitectónico. Una de las tantas consecuencias de este terrible acontecimiento consistió en que el Gobierno colombiano rompió relaciones con la Unión Soviética. Lo anterior resume la situación social, política y económica que se vivió en Colombia entre 1946 y 1948.

Para responder al segundo interrogante del objeto de estudio, inicio con el abogado Jorge Soto del Corral, quien administró la Alcaldía de Bogotá de marzo a noviembre de 1944. Durante su gobierno se implementó el plan Soto-Bateman, más conocido como el de la primera zonificación de Bogotá. Este plan de urbanismo estaba basado en un sistema de valorización amparado por la Ley $1 .{ }^{a}$ de 1943 , e incluía, además de una zonificación, el diseño y la construcción de vías en la capital de la república. Se trataba de descongestionar el centro de la ciudad y conectarlo de manera más eficaz con sus sectores circundantes. Desde el momento en que el plan Soto-Bateman fue estudiado por un comité técnico de la Sociedad Colombiana de Arquitectos (SCA) se propuso la opción de crear un departamento de planeación municipal que se encargara de manera autónoma de controlar el desarrollo urbanístico de 
Bogotá. La SCA presentó como resultado de su estudio un plan alternativo consistente en el desarrollo y ampliación de la malla vial para realizar conexiones en los ejes norte-sur y centro-occidente. En el sentido norte-sur se propusieron varias vías, entre las que se destacan la de la carrera $4 .^{\text {a }}$, que no se realizó, y la carrera 9. ${ }^{a}$, que se convirtió posteriormente en la ampliación de la carrera 10. ${ }^{\text {a }}$ Se planteó también ampliar las carreras 7.a y 13 a partir de la calle 26 , prolongar la avenida $\mathrm{Ca}-$ racas hacia el sur desde la carrera 1. y hacia el occidente se propuso la creación de la avenida de las Américas y la avenida de los Comuneros. La avenida de las Américas se ideó con la finalidad de descongestionar el tránsito de la capital hacia los municipios del occidente y "hacer que las nuevas urbanizaciones se orienten nuevamente hacia el río y no sigan dispersándose a lo largo de las estribaciones de Monserrate y Guadalupe" (Semana, 1947, pp. 16-19); su diseño fue atribuido a los arquitectos Herbert Rittter y Carlos Martínez, y se trataba de una amplia vía de 114 metros entre sus parámetros, "con una longitud total de 6,5 kilómetros contados desde el cruce de las calles 26 y 34 , prolongados hasta el parque de estacionamiento del aeropuerto de Techo" (Ritter, 1946, p. 7). Los $6,5 \mathrm{~km}$ de la vía estaban divididos en dos tramos. En el aeropuerto de Techo comenzaba el primero y conectaba con Puente Aranda; su longitud era de 3,5 kilómetros y consistía en "dos calzadas laterales de seis metros de ancho, diseñadas para facilitar el tránsito de los viajeros y el acceso a las propiedades riber[eñ] as" (Ritter, 1946, p. 7). La amplia zona comprendida entre las dos calzadas se propuso inicialmente para "campos populares de deportes: football, tennis, basket ball y pistas para jinetes y ciclistas" [sic] (Ritter, 1946, p. 7). El emplazamiento del monumento a las banderas le correspondió a este primer sector de la obra, del aeropuerto de Techo a Puente Aranda; en su centro se destinó la construcción del monumento, en medio de una glorieta, alrededor de la cual se dispusieron espacios para el parqueo de vehículos.

El segundo sector correspondía a la avenida de las Américas, consistente en $3 \mathrm{~km}$ desde Puente Aranda hasta el cruce de las calles 26 y 34. Albergaba dos calzadas exteriores de $6 \mathrm{~m}$ y dos vías principales de $9 \mathrm{~m}$ de ancho, que podían recibir cada una "tres filas de vehículos" 
(Ritter, 1946, p. 7). Esta tipología daba como resultado una vía rápida, razón por la que se determina la prohibición de tránsito de "ganados, aves, reses conducidas por pastores o por jineteros" (Ritter, 1946, p. 7) y vehículos de tracción animal. La avenida de las Américas se constituyó en la primera cuyo trazado no fue circunscrito a un camino preexistente, como se puede verificar en cualquier plano de la ciudad con fecha de 1944; de acuerdo con esto, se puede señalar que esta fue la primera vía concebida conforme a diseños de ciudad moderna. La propuesta para la construcción de esta avenida se originó con motivo de la celebración de la IX Conferencia Panamericana, puesto que en el Acuerdo 45 de 1946, expedido por el Concejo de Bogotá, se dispuso su ejecución en concordancia con el siguiente texto:

Dispónese la ampliación y regulación de la calle 13 en el trayecto comprendido entre la carrera 13 y Puente Aranda, en la intersección con la avenida de las Américas, de acuerdo con los planes y proyectos de la Secretaría de Obras Públicas Municipales.

El conjunto escultórico albergado en la glorieta fue terminado en 1948, pero a causa de los acontecimientos del 9 de abril de ese año no pudo ser inaugurado. La obra le fue encargada al escultor Alonso Neira Martínez por el líder conservador Laureano Gómez, quien era el presidente de la Comisión de la IX Conferencia Panamericana.

Se destacan los siguientes aspectos biográficos del escultor Neira Martínez, quien fue alumno y profesor en la Escuela de Bellas Artes. Su nombre sobresale en la historia del arte colombiano como uno de los mayores exponentes de la escuela academicista. Participó en el Salón de Artistas Colombianos de 1940 con las obras Cabeza de hombre, talla directa en madera, y Cabeza de mujer, cerámica en bizcocho. En el Salón de 1942, el artista obtuvo el Primer Premio en Escultura por sus obras Vida y Reposo, ambas realizadas en terracota. Dos años después, en el V Salón, obtuvo Medalla de Oro por su obra Desnudo. Su obra pública más destacada es el monumento a las banderas, en la avenida de las Américas, en Bogotá (Ortega, 1965, p. 323).

Según entrevista realizada por el autor de este escrito al hijo del escultor, el arquitecto Germán Neira, miembro de la SCA, en noviembre 12 de 2011, Laureano Gómez conocía al escultor Ramón Barba y 
deseaba que fuera él quien creara el monumento, pero Barba se encontraba muy ocupado y referenció al escultor Neira para que hiciera el trazado arquitectónico de la glorieta, al igual que el esquema escultórico. El líder conservador supervisó directamente el proceso de diseño y talla de las mujeres desnudas que comprenden el conjunto. Las esculturas femeninas fueron trabajadas al vacío en láminas de arcilla de 3 y $4 \mathrm{~cm}$ y, de acuerdo con el proceso de desarrollo de su talla, se utilizaron al final láminas de medio centímetro para ajustar los detalles. El escultor Neira trabajaba con modelos y pudo haber creado la escultura de su mujer ideal a partir de las partes de los cuerpos de las mujeres que le servían en calidad de modelos. El diseño final de la escultura femenina corresponde al gusto estético personal del artista en cuanto a su ideal de mujer. Las esculturas fueron medidas en noviembre 15 de 2011 por la artista plástica Cristina Salazar, egresada de la Escuela de Bellas Artes de la Universidad Nacional, quien determinó una altura de $1,90 \mathrm{~cm}$, medida estándar para todas las mujeres de la obra. No se pudieron obtener las medidas del contorno total por cuanto las esculturas se encuentran pegadas al cilindro de base; sin embargo, estas corresponden a $52 \mathrm{~cm}$ de busto, $40 \mathrm{~cm}$ de cintura y $52 \mathrm{~cm}$ de cadera. Si se pudiera medir el total de la circunferencia del cuerpo femenino, por aproximación estas medidas darían un total de más de 90, 60, 90 , las que corresponderían a tallas normales ideales. Hoy en día, este es el prototipo de anatomía de las mujeres que concursan en los reinados de belleza, por lo que se podría decir que el escultor Neira estaba adelantado a su tiempo en cuanto a un ideal de fisiología femenina. De esta primera escultura resultante se realizaron seis especímenes con pequeñas variaciones, y a cada una de ellas se les otorgó un elemento iconográfico que representaría los problemas que en ese momento aquejaban a América Latina. En virtud de lo anterior, el escultor Neira representa a la ciencia por medio de la mujer que sostiene en su mano izquierda, a la altura de la cintura, el símbolo $\pi$; el comercio es simbolizado por el caduceo de Hermes, el cual es sostenido por la mano derecha de la mujer a la altura de sus senos; la mujer que simboliza la justicia sostiene una espada a la altura del vientre, con la mano izquierda colocada sobre la 
punta del mango, que por debajo de este se apoya con la mano derecha, y la punta de la espada está apoyada sobre el suelo; la mujer representante de la agricultura tiene un espécimen del maíz con una altura desde el suelo hasta más arriba de sus senos, mantenido con la mano derecha abajo y con la mano izquierda arriba; la sabiduría está representada por una mujer que sustenta en su mano izquierda un pergamino enrollado con una hoja de laurel sobrepuesta, situado justo debajo de su seno izquierdo; el progreso lo representa la mujer que coloca la mano izquierda a la altura del muslo y con la mano derecha exhibe la punta de un rayo, este símbolo se identifica con la electricidad.

De estas seis mujeres con su respectiva iconografía el artista diseñó veinte conjuntos en total, colocados sobre una base en forma de circunferencia que se alza hacia el cielo en un ángulo de $22,5^{\circ}$ aproximadamente, y que se cierra en su diámetro una tercera parte; sobre esa superficie fijó las seis mujeres alrededor de unos tallos de acanto, con clara influencia del Art nouveau, y en el centro de estos acantos se instalaron unos mástiles, en los que se izarían cada una de las banderas de los países participantes en la conferencia.

Estos conjuntos de seis mujeres fueron emplazados en la parte externa de la circunferencia que forma la glorieta, en cuyo centro se levanta un nivel superior en un ángulo de $15^{\circ}$, el cual se alza hasta completar una altura de $1 \mathrm{~m}$ (ambas medias aproximadas) y en su centro también fue colocado un mástil rodeado por los escudos forjados en bronce de los 14 departamentos que conformaban el país, los cuales fueron robados en esa época y, por esta razón, se reemplazaron por una fuente.

Si se pregunta la razón por la cual el escultor Neira decidió realizar unas esculturas de mujeres desnudas, conforme a la influencia de la escuela neoclásica, para el monumento a las banderas, la respuesta se puede indagar desde el siglo XIX, puesto que en las clases de la Escuela de Bellas Artes predominaban los estudios anatómicos a partir de yesos. Es sabido que en 1893 Epifanio Garay, en su actuar como director de dicha escuela, promovió estudios con modelo al natural y, de acuerdo con sus influencias académicas obtenidas en la Académie Julien de París, presentó en el Salón de 
1899 la obra La mujer del levita de los montes de Efraim, trabajo realizado con modelo. La Cátedra de Dibujo Académico fue establecida en la Escuela de Bellas Artes en los primeros años del siglo XX y los primeros premios en esta categoría los disputaron en 1904 y 1905 Coroliano Leudo y Domingo Moreno Otero. Posteriormente, Andrés de Santa María, director de la escuela entre 1904 y 1911, también impulsó entre sus profesores y estudiantes el trabajo académico con modelo al desnudo. En la Exposición del Centenario (1910), en el pabellón de escultura, predominaron los bustos, así como la escultura conmemorativa, y en la sección de pintura se destacaron los paisajes, el retrato y el bodegón. En 1926 Laureano Gómez, en su calidad de ministro de Obras Públicas en la presidencia de Pedro Nel Ospina, ordenó obras de adecuación para el parque del Centenario, por tal motivo se construyó una fuente que debía ser complementada por una escultura, y la obra que finalmente se escogió como motivo central fue La Rebeca, escultura de tipo neoclásico que se le atribuye al escultor Roberto Henao Buriticá (1898-1964). Esta obra causó polémica en su momento por tratarse de un desnudo femenino, razón por la que se le rodeó de plantas acuáticas como propósito ornamental y para esconder su desnudez. En las décadas de los treinta y cuarenta predominaron estas temáticas en la producción artística nacional y se mantuvieron los estudios con modelo en la Escuela de Bellas Artes.

Alonso Neira ingresó a la Escuela de Bellas Artes en 1933 y tuvo de profesores al escultor Ramón Barba, quien dictó el Curso Escultura al Natural a Jose Domingo Rodríguez, Gustavo Arcila Uribe y Domingo Moreno Otero, entre otros. En el Salón de 1931, con El solitario, trabajo de influencia neoclásica realizado en cerámica vidriada por Gustavo Arcila, obtuvo el Segundo Premio en Escultura. En la década de los cuarenta se presentó un gran desarrollo de obras con temáticas relacionadas al desnudo, como se puede observar en la entrega de premios del III Salón de Artistas Colombianos, en 1942, en el que la obra de José Domingo Rodríguez, titulada Angustia, fue galardonada con el Primer Premio en Escultura; a La Anunciación, de Carlos Correa, se le retiró el Primer Premio en Pintura; Alonso Neira obtuvo Medalla de Oro por su escultura de desnudo femenino llamada Reposo 
y Alicia Cajiao Medalla de Bronce por su óleo La negra Simona. En el V Salón de Artistas Colombianos, en 1944, Miguel Díaz Vargas logró un premio de estímulo con su obra Estudio en gris, la cual representa una dama escasamente cubierta por una tela, y fueron premiados con sendos galardones de estímulo los escultores Alonso Neira por su obra escultórica Desnudo y Miguel Sopó por Maternidad, que presenta a una madre con su hija en estado de desnudez.

Acerca de las razones que motivaron a Alonso Neira para realizar unos desnudos escultóricos en el monumento a las banderas, estas están claramente definidas, primero, por las asignaturas que cursó en la Escuela de Bellas Artes; segundo, por las enseñanzas e influencias de las obras creadas por sus profesores; tercero, por el auge de la temática del desnudo en pintores y escultores; cuarto, por los premios que se otorgaban a este tipo de obras en los salones de artistas colombianos; quinto, porque el neoclasicismo era la escuela escultórica a la que pertenecía Alonso Neira; sexto, porque al escultor Neira le gustaba trabajar el desnudo; por último, porque probablemente Laureano Gómez se identificó con la corriente artística neoclásica y con la temática desnudista profesada por el maestro Neira, que se asimilaba en escuela y en tema a la escultura de La Rebeca, contratada por el líder conservador cuando era ministro de Obras Públicas. Probablemente, por esto Laureano se identificó tanto con las esculturas de las mujeres realizadas por el maestro Neira que supervisó su proceso de creación de manera personal y semanal en el taller momentáneo del maestro Neira, localizado en el antiguo edificio de los Ministerios, al lado del palacio de Nariño y al occidente de la iglesia de San Agustín, pues dicho edificio se encontraba vacío en ese entonces $y$ fue prestado para tal fin por el entonces ministro Gómez.

Para concluir este ensayo, voy a contextualizar y a relacionar con el conjunto escultórico de Neira algunos pensamientos expuestos por A. Remesar en su texto Hacia una teoría del arte público.

Sobre el arte público Remesar presenta ideas comunes de los autores Mitchel (1992), Miles (1989) y Raven (1989), las cuales identifico claramente con el origen y concepción del monumento a las banderas:

La identificación tradicional del arte público con la escultura pública 
o el ornato público; la identificación entre estatua y monumento; la emergencia de la obra en lo que denominan espacio público, y la existencia previa del arte que, en todo caso, se aplica al espacio público (Remesar, 1997, p. 26).

El monumento a las banderas se puede considerar una clase de arte, solicitado, costeado y de propiedad del distrito capital. Su origen tiene que ver con una propuesta conjunta en los ámbitos de gobierno distrital y nacional; el monumento se diseñó en un espacio que estaba disponible y constituyó un punto de referencia en el paisaje urbano, más aún, hoy en día la estación de Transmilenio circunvecina al conjunto escultórico lleva el nombre Banderas, lo que produce una sensación de lugar y conecta la localidad con otros puntos estratégicos, lo que se denomina red local, y cumple uno de los objetivos de los arquitectos Herbert Rittter y Carlos Martínez en el trazado de la avenida de la Américas y de la razón de ser de la glorieta. El conjunto escultórico albergado allí tenía varias funciones: embellecer, conmemorar y rendir un homenaje a los representantes de los países asistentes a la IX Conferencia Panamericana. Considero de importancia subrayar que los dignatarios extranjeros tuvieron la oportunidad de admirar la obra durante su recorrido del aeropuerto de Techo al Capitolio Nacional y de regreso al aeropuerto desde el norte de la ciudad, donde sesionaban y se alojaban.

El cuerpo humano, uno de los referentes creativos del escultor Alonso Neira, utilizado como vehículo de representación visual y de imitación de la naturaleza ajustada a la emulación de las formas corporales creadas como resultado de su huella o trazo, pudo haber consistido en la representación de la pulsión (energía traducida en un objeto del deseo), en virtud del yo concebido culturalmente desde el siglo XVII. La representación escultórica de la figura humana, ideada conforme a un sistema de pulsiones, puede también relacionarse con los objetos que en ellas se soportan, como es el caso de las figuras femeninas que se encuentran adheridas a una planta de acanto, en cuyo tallo se yergue un mástil con el objetivo de sostener las banderas, sistema de signos que busca acentuar el volumen simbolista del ser humano.

$\mathrm{El}$ arte del cuerpo, resultado de su elaboración como lenguaje artístico, actúa al igual que un sistema sensorial complejo que genera motivaciones por medio de los sentidos, 
puesto que su contemplación no requiere el uso de la metáfora y el cuerpo como presencia plástica se constituye en un campo muy valioso para el desarrollo del arte público.

Sobre la referencia espacial Neira tuvo potestades totales para diseñar y construir el monumento escultórico en el espacio de la circunferencia o glorieta; gozó del privilegio de cubrir todos los puntos cardinales con sus esculturas de mujeres, puesto que envolvían también las coordenadas corporales, las cuales también se sitúan en correspondencia con el cielo, por el tallo de las plantas de acanto, así como por las astas y por la dirección de la actividad circundante, el paso de los automóviles y de los ciudadanos. Su planteamiento y su objetivo contemplativo como obra de arte público fue el resultado de la organización del territorio,ya que la admiración del cuerpo como procedimiento referente espacial supone por necesidad la observación de este como agente en el espacio, móvil que organiza la zona con el movimiento.

El conjunto escultórico denominado Las banderas panamericanas se constituye en arte público monumental y refleja valores estéticos, estilísticos, sociales y simbólicos. Cumple con todas las funciones que le son inherentes:

[...] conmemora, mejora el espacio visual, ayuda a la regeneración económica de la zona mediante el turismo y la inversión, ayuda a la regeneración cultural y artística para identificar una comunidad, ayuda a la gente para administrar el espacio público y a contestar una política más general sobre la calidad de vida (Remesar, 1997, pp. 48-49).

\section{Referencias}

AA. VV. (2010). Vuelo al pasado. Bogotá: IGAC y Villegas.

Vásquez, W. (2008). Escuela Nacional de Bellas Artes de Colombia. Tesis de maestría. Universidad Nacional de Colombia, Bogotá.

Pombo, L. et al. (2008). Bogotá, un museo a cielo abierto. Guía de esculturas y monumentos conmemorativos en el espacio público (vol. 1). Bogotá: Alcaldía Mayor, Instituto Distrital de Patrimonio Cultural.

Fundación Erigae y Rodríguez, W. (2006). Corporación La Candelaria. Atlas histórico de Bogotá, 19111948. Bogotá: Nomos.

Saldarriaga, A. (2000). Bogotá siglo XX. Urbanismo, arquitectura y vida 
urbana. Bogotá: Departamento Ad- Ortega, C. (1965). Diccionario de ministrativo de Planeación Distrital. artistas. Bogotá: Tercer Mundo.

Garavito, F. y Santos, E. (1994). Bo- $\quad$ Ritter, H. (1946). La avenida de las gotá desde el aire. Bogotá: Villegas. Américas. Proa, 1, 7.

Meiseles, A. (1992). Senderos. Bogotá: Cultural. 


\section{Anexo}

Figura 1. Aeropuerto de Techo, glorieta de Banderas, avenida de las Américas, humedal de Techo (1948)

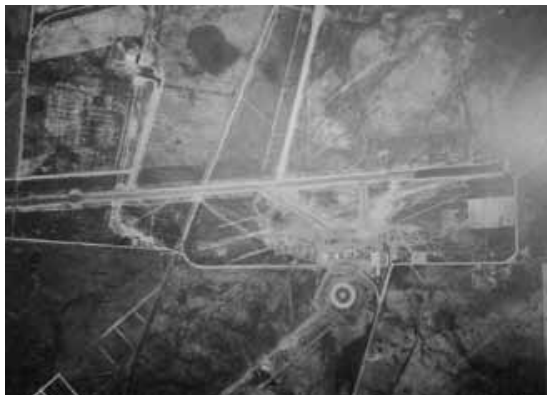

Fuente: AA. W. (2010). Vuelo al pasado (p. 81). Bogotá: IGAC y Villegas

Figura 2. Avenida de las Américas y avenida 28 (1947)

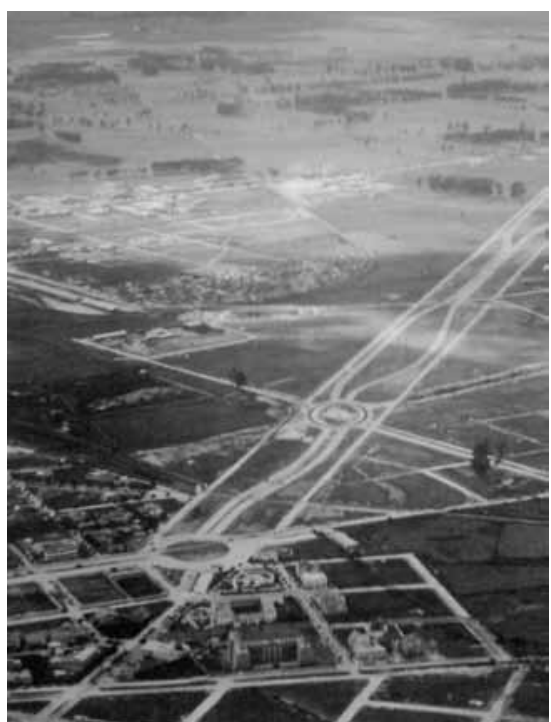

Fuente: AA. W. (2010). Vuelo al pasado (p. 54). Bogotá: IGAC y Villegas
Figura 3. Las banderas de las veintiún naciones americanas, izadas en la avenida de las Américas (1948).

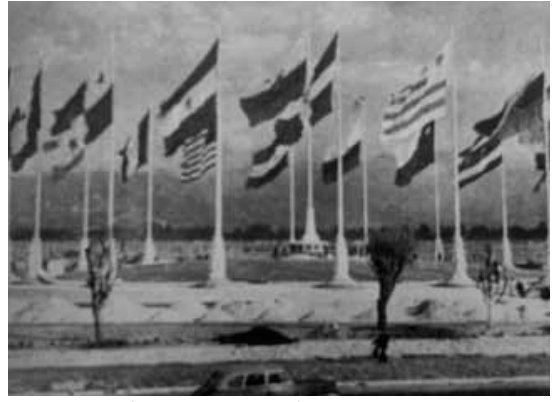

Fuente: Fundación Erigae y Rodríguez, W. (2006)

Corporación La Candelaria. Atlas histórico de Bogotá, 1911 . 1948 (p. 529). Bogotá: Nomos.

Figura 4. Glorieta de las Banderas en la avenida de las Américas (1948)

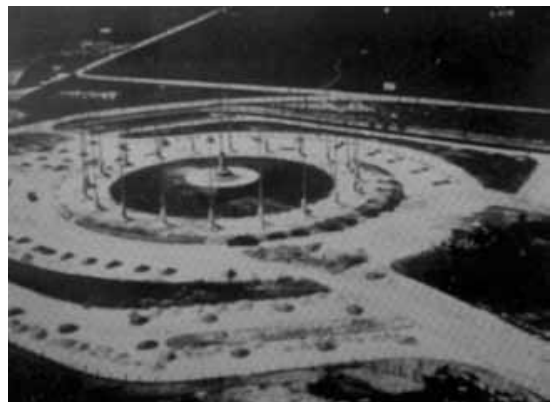

Fuente: Fundación Erigae y Rodríguez, W. (2006).

Corporación La Candelaria. Atlas histórico de Bogotá, 1911 1948 (p. 528). Bogotá: Nomos 
Figura 5. monumento a las Banderas (2010)

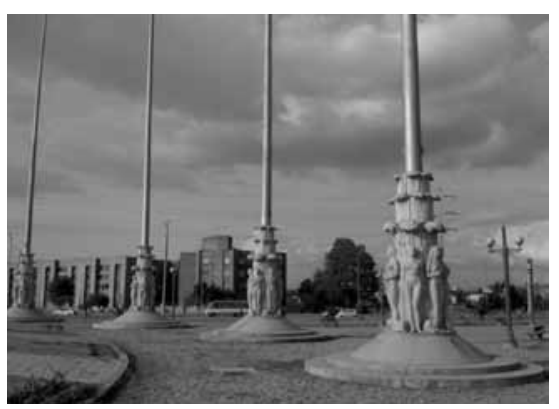

Fuente: Archivo particular

Figura 6. monumento a las Banderas (2010)

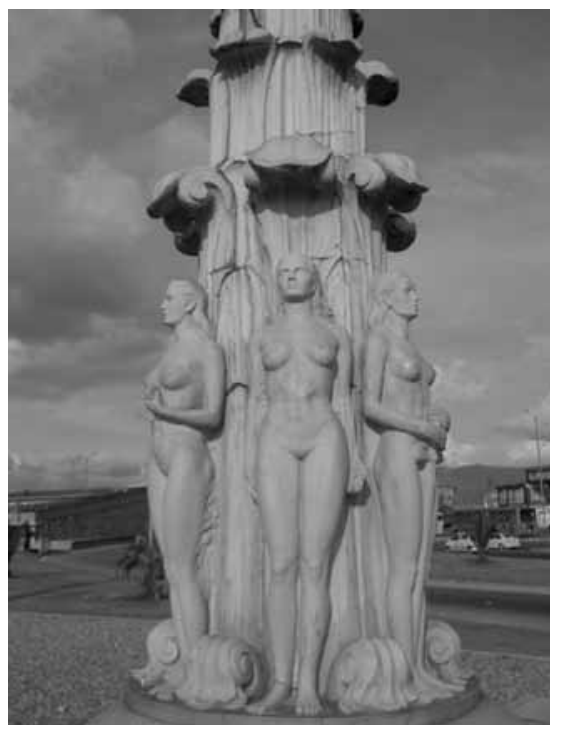

Fuente: Archivo particular
Figuras 7 y 8 . monumento a las Banderas (2011)
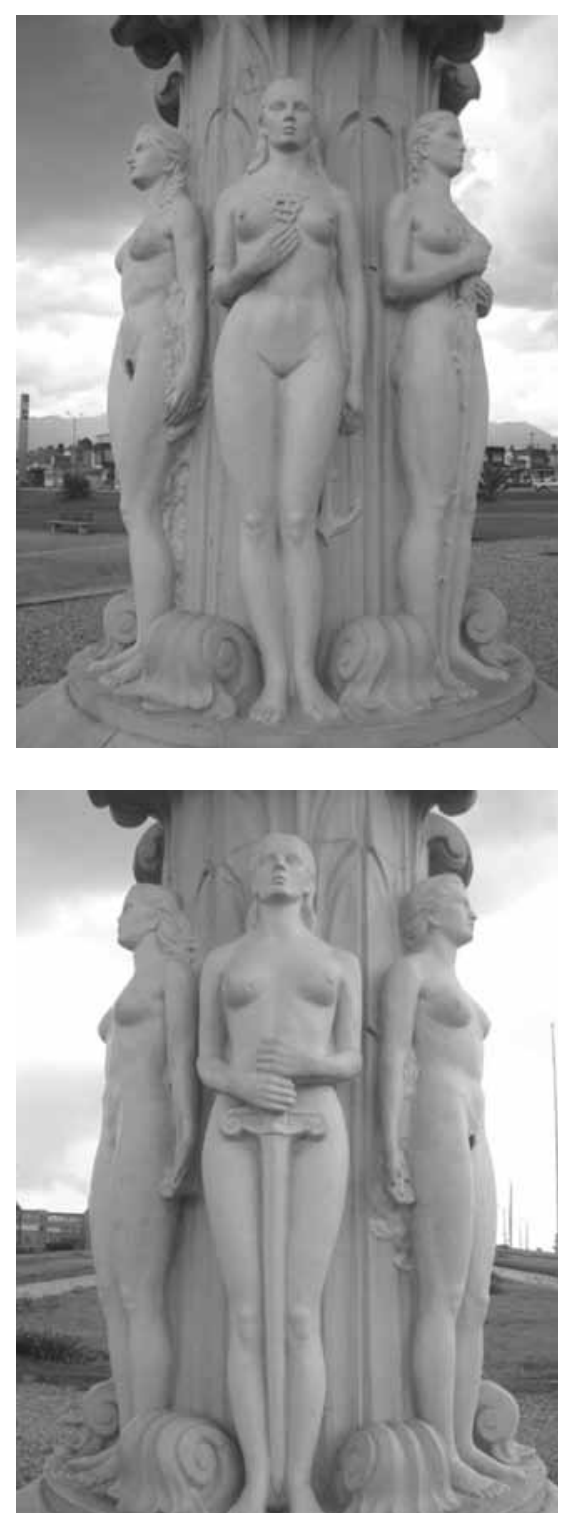

Fuente: Archivo particular 
Figuras 9 y 10. monumento

a las Banderas (2011)
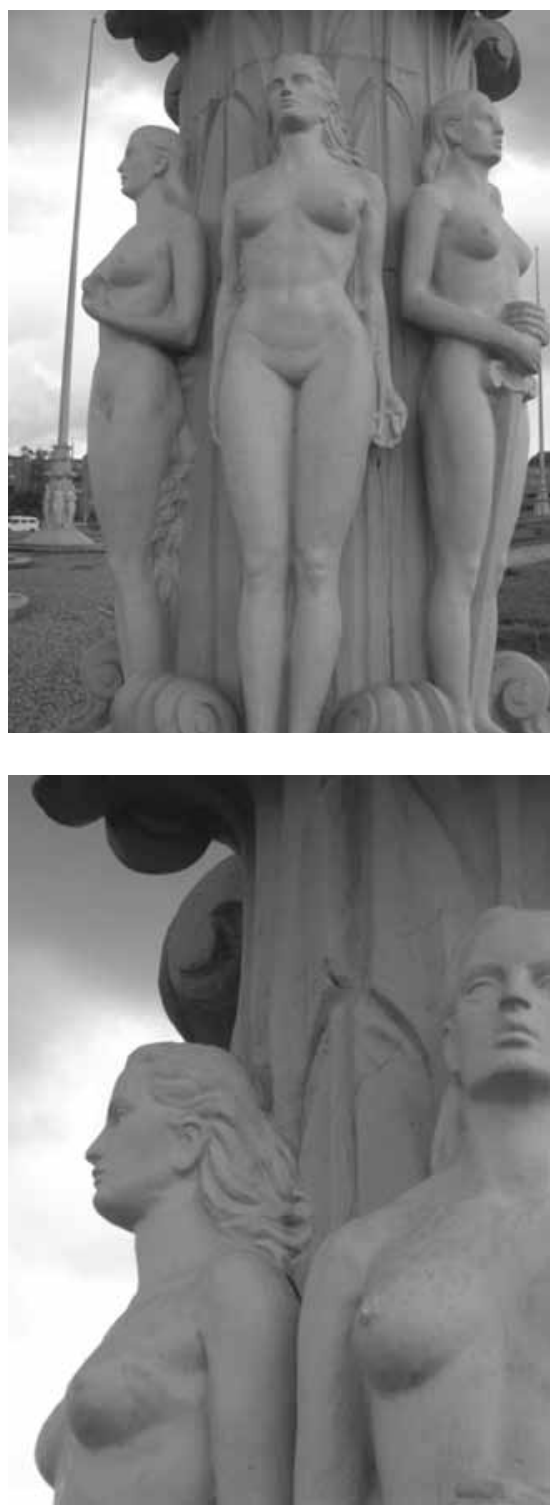

Fuente: Archivo particular
Figuras 11 y 12. monumento

a las Banderas (2011)
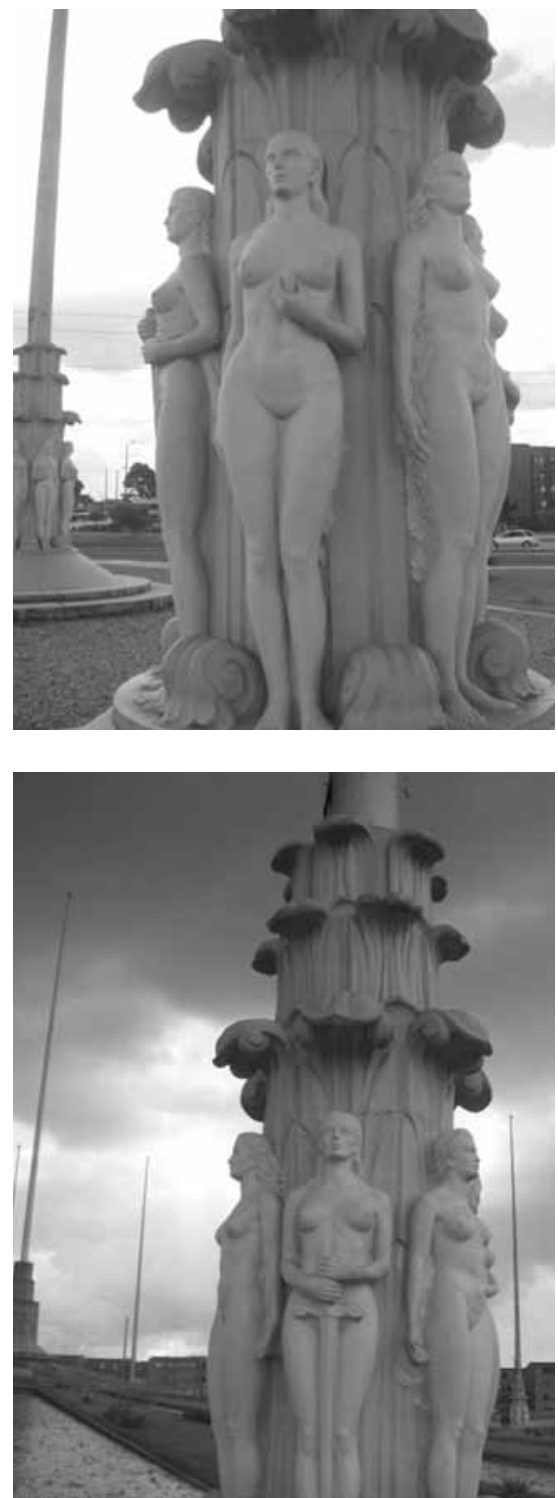

Fuente: Archivo particular 
- El Monumento a las Banderas en la avenida las Américas en Bogotá - Diego Carrizosa

Figuras 13 y 14. monumento a las Banderas (2011)
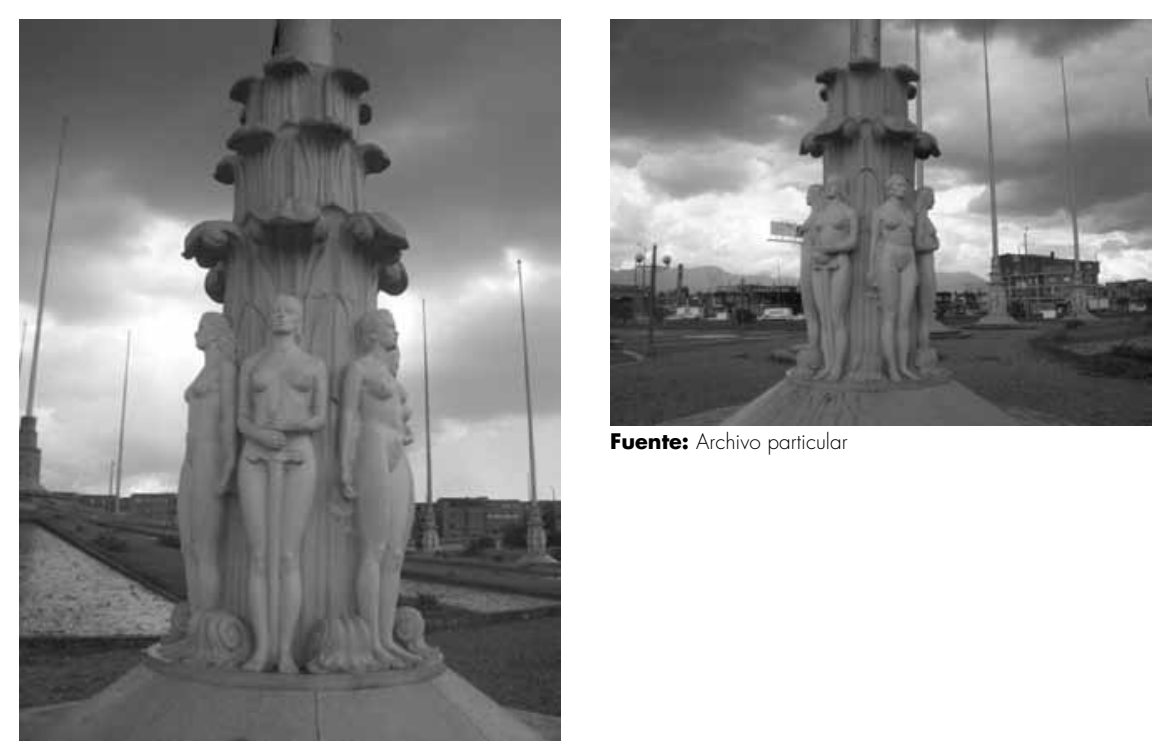

Fuente: Archivo particular 